ИЗВЕСТИЯ АКАДЕМИИ НАУК ЭСТОНСКОИ ССР. ФИЗИКА - МАТЕМАТИКА PROCEEDINGS OF THE ACADEMY OF SCIENCES OF THE ESTONIAN SSR. PHYSICS * MATHEMATICS

$1987,36,3$

удК 621.314 .63

T. $\mathrm{TOMCOH}$

\title{
ИДЕАЛИЗИРОВАННАЯ СИСТЕМА ВЫПРЯМИТЕЛЕЙ НА ОБЩИХ ПРОИЗВОЛЬНО-УПРАВЛЯЕМЫХ ТИРИСТОРАХ
}

\author{
(Представил И. Эпик)
}

Энергетические свойства тиристорного моста при его несимметричном управлении рассмотрены в $\left[{ }^{1}\right]$. Наряду с повышенным коэффициентом сдвига несимметрично-управляемый мост обладает увеличенным риском коммутационных отказов, так как в нем нельзя применять дублированные через $\pi / 3$ управляющие импульсы. Однако устройство $\left[{ }^{2}\right]$ обеспечивает надежное управление короткими управляющими импульсами (несимметричного) мостового преобразователя и позволяет реализовать системы автономных выпрямителей с общим трансформатором и с общей произвольно-управляемой группой тиристоров. Эта система осуществлена с помощью двух выпрямителей (рис. 1), автономно-управляемые тиристорные группы которых названы тиристорными регуляторами (ТР). Нами показано, что данная система имеет некоторые преимущества перед ранее описанной системой $\left[{ }^{3}\right]$, общие тиристоры которой управлялись по минимальному углу из управляющих ТР-импульсов. Под произвольностью понимаем то обстоятельство, что (на стадии исследования) угол управления общими тиристорами не связан с управляющими ТР-импульсами $\alpha^{\prime}, \alpha^{\prime \prime}$. Идеализация системы заключается (аналогично $\left.\left[{ }^{3}\right]\right)$ в следующих упрощающих предпосылках:

1) сеть является симметричной и гармонической;

2) индуктивность в цепи нагрузки бесконечно большая $L_{d} \rightarrow \infty$, т. е. выпрямитель работает в режиме непрерывного тока $I_{d}(\omega t)=\mathrm{const}$;

3) пренебрегаем процессом коммутаций $\gamma \rightarrow 0$;

4) считаем токи нагрузки равными $I^{\prime}{ }_{d}=I_{d}^{\prime \prime}=1$, т. е. проведем анализ в относительных единицах.

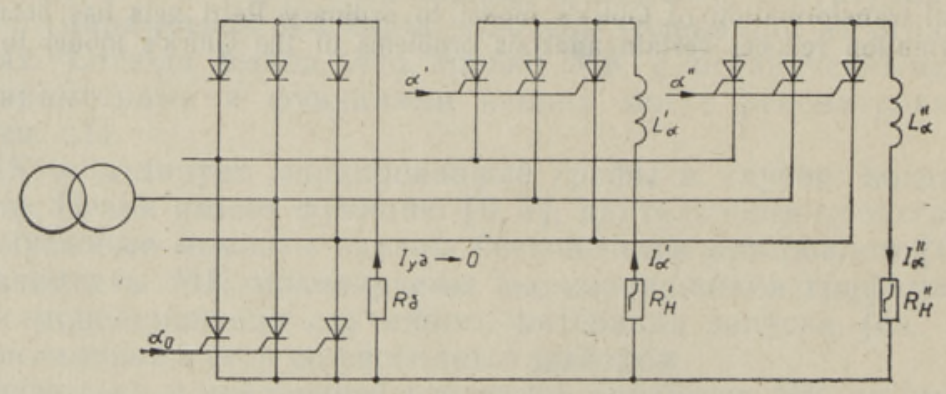

Рис. 1. Принципиальная схема системы управляемых выпрямителей с общей произвольно-управляемой тиристорной группой. 
С̆стема упрощена до двух ТР, что есть минимально необходимое йх число для образования системы. Нулевые диоды не применены, поскольку, по $\left[{ }^{3}\right]$, это сводится к ограничению функциональных возможностей системы. Вспомогательные диоды загружаются малым током удержания общих тиристоров $I_{\text {уд }} \rightarrow 0$.

Выходное напряжение ТР зависит от угла его управления $\alpha \in$ $\in\left\{\alpha^{\prime}, \alpha^{\prime \prime}\right\}$ и от угла управления общей тиристорной группы $\alpha_{0}$

$$
U_{d} / U_{d 0}=\left(\cos \alpha+\cos \alpha_{0}\right) / 2 .
$$

Здесь $U_{d 0}$ - выходное напряжение неуправляемого (диодного) мостового выпрямителя. Отсюда следуют ограничения по максимально возможному напряжению выпрямительного режима

$$
\max U_{d} / U_{d 0}=\left(\cos \alpha_{0}+1\right) / 2
$$

и по минимальному напряжению инверторного режима

$$
\min U_{d} / U_{d 0}=\left(\cos \alpha_{0}-1\right) / 2 .
$$

Ограничение (3) приведено без учета запаса (на коммутацию и время восстановления вентилей). Для достижения равного с симметричным мостовым выпрямителем диапазона регулирования по выходному напряжению должна быть повышена устанавливаемая мощность $P^{*}$ ном преобразовательного трансформатора

$$
P_{\text {ном }}^{*} / P_{\text {ном }}=2 /\left(1+\cos \alpha_{0}\right),
$$

где $P_{\text {ном }}$ - устанавливаемая мощность трансформатора для симметричного моста. Это обстоятельство является определенным недостатком несимметричного управления, не отмеченным в [ $\left.{ }^{1}\right]$.

В идеальной системе (по рис. 1) при «идеальных» СИФУ, не чувствительных к коммутационным помехам соседнего ТР, оба выходных напряжения взаимно инвариантны: $U_{d}^{\prime \prime} \neq f\left(U_{d}^{\prime}\right), U_{d}^{\prime} \neq f\left(U_{d}^{\prime \prime}\right)$. Это свидетельствует о явном преимуществе системы в сравнении с системой, приведенной в $\left[{ }^{3}\right]$, где для инвариантного управления требуются специальные корригирующие устройства.

Рассмотрим свойства ТР, отличные от свойств симметричного мостового выпрямителя. Выходное напряжение ТР содержит при $\alpha \neq \alpha_{0}$ составляющую с частотою 150 Гц. Его расчетные соотношения найдены в $\left[{ }^{4}\right]$ для случая «ведомого тиристорного регулятора в системе с общей тиристорной группой», так как для формирования спектра выходного напряжения безразличны функциональные причины несимметричного управления. По $\left[{ }^{4}\right]$, коэффициенты Фурье выражаются для составляющей, частотой 150 Гц, следующим образом:

$$
\begin{aligned}
& a_{2}=3\left\{\cos 4 \alpha_{0}-\cos 4 \alpha-2\left[\cos 2 \alpha_{0}-\cos 2 \alpha\right]\right\} / 8 \pi, \\
& b_{2}=3\left\{\sin 4 \alpha_{0}-\sin 4 \alpha-2\left[\sin 2 \alpha_{0}-\sin 2 \alpha\right]\right\} / 8 \pi,
\end{aligned}
$$

откуда можно получить формулу для расчета комплексного коэффициента Фурье

$$
\begin{gathered}
c_{2}=\sqrt[3]{10-4\left[\cos 2 \alpha_{0} \pm \cos 2\left(\alpha_{0}+\Delta_{\alpha}\right)-\cos 2\left(\alpha_{0} \pm 2 \Delta_{\alpha}\right) \pm \cos 2\left(\alpha_{0}-\Delta_{\alpha}\right)-\right.} \\
\cdots-8 \cos 2 \Delta_{\alpha}-2 \cos 4 \Delta_{\alpha} / 8 \pi .
\end{gathered}
$$

В (5) верхние знаки действуют для запаздывающего регулятора $\alpha^{\prime \prime}=$ $=\alpha_{0}+\Delta_{\alpha}$, а нижние - для опережающего регулятора $\alpha^{\prime}=\alpha_{0}-\Delta_{\alpha}$. Расчитанная по (5) функциональная зависимость $c_{2}=f\left(\alpha_{0}, \Delta_{\alpha}\right)$, показанная на рис. 2 , пригодна для расчета $L_{d}$ фильтра, если данная система применяется для питания многопостовой электродуговой установки. Среди 


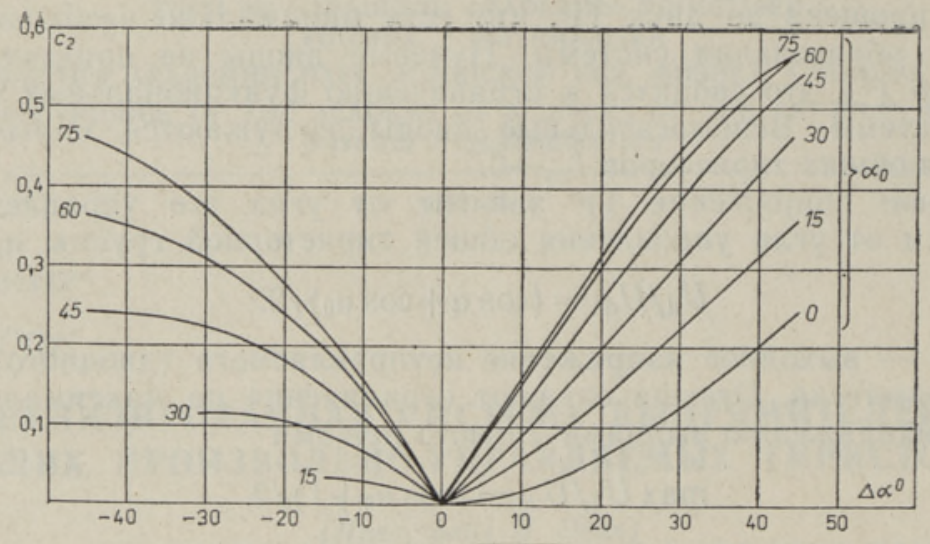

Рис. 2. Комплексный коэффициент Фурье составляющей 150 Гц выходного напряжения тиристорного регулятора.

дуговых нагрузок встречаются такие, которые характеризуются малым значением дифференциального сопротивления. Например, т. н. вакуумная электрическая дуга или плазмотрон с межэлектродными вставками допускают изменение тока в широком диапазоне без существенного изменения напряжения. Следовательно, системы электропитания подобных нагрузок могут быть спроектированы для постоянного угла $\alpha_{0}=$ $=$ const, $\Delta_{\alpha} \rightarrow 0$. Проиллюстрируем результаты анализа следующими примерами.

I. Задана система электропитания с общей диодной группой, которая работает в режиме $U_{d}^{\prime} / U_{d 0}=U_{d}{ }^{\prime \prime} / U_{d 0}=0,5$, что характерно для источников питания электродуговых нагрузок. Это обеспечивается при $\alpha^{\prime}=\alpha^{\prime \prime}=90^{\circ}$, при котором $c_{2} \approx 0,48$.
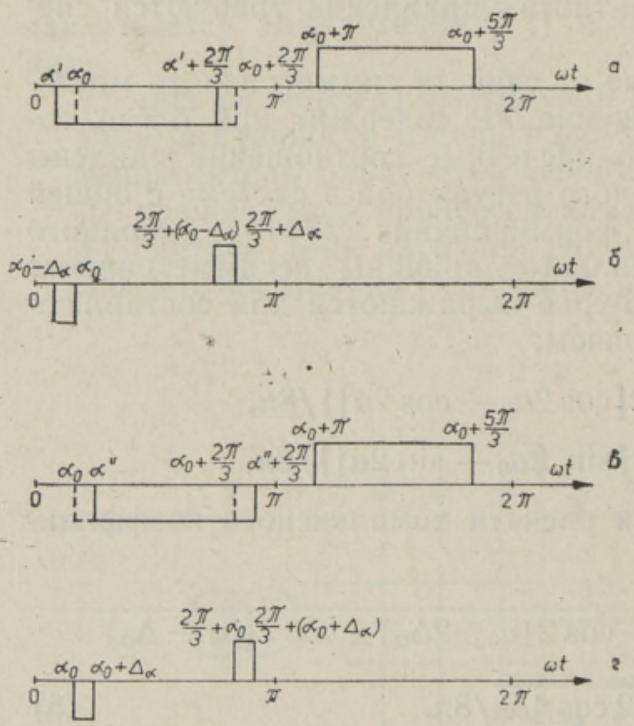

Рис. 3. Эпюры входного тока системы: $a$ - для ТР с опережающим углом управления, $в-$ для ТР с запаздывающим углом управления, б и а - соответствующие сигналы «по-

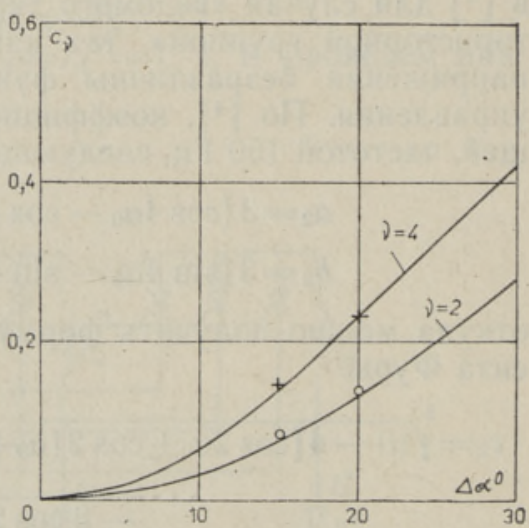

Рис. 4. Комплексный коэффициент Фурье входного тока системы управляемых выпрямителей с общей произвольно-управляемой тиристорной группой. 
II. Задана система электропнтания с общей тиристорной группой, при неравных напряжениях $U_{d}^{\prime} / U_{d 0}=0,6, \quad \alpha^{\prime}=46^{\circ}$ и $\quad U_{d}^{\prime} / U_{d 0}=0,4$, $\alpha^{\prime \prime}=72$ при выборе $\alpha_{0}=59^{\circ}=$ const. Таким образом, $\Delta_{\alpha}= \pm 13^{\circ}$. По рис. 2 установим для первого ТP $c_{2}^{\prime}=0,175$, а для второго ТР $c_{2}^{\prime \prime}=0,19$. Пример даже со значительным отличием выходных напряжений в системе показывает существенное уменьшение в выходном напряжении составляющей 150 Гц.

Входной ток каждого несимметричного выпрямителя содержит четные гармонические составляющие, из которых рассмотрим поведение наиболее существенных - 2-го и 4-го порядков.

По цепному правилу $\left[{ }^{5}\right]$, анализу следует подвергать сигнал «помехи», который отличает входный ток ТР от соответствующего тока симметричного моста при $\alpha_{0}$ (эпюры сигналов, на которых пунктиром обозначена кривая тока симметричного моста, не содержащего 2-го и 4-го гармонических составляющих, приведены на рис. 3).

Выберем угол управления общей группы таким образом, чтобы было удовлетворено условие $\alpha_{0}-\alpha^{\prime}=\alpha^{\prime \prime}-\alpha_{0}=\Delta_{\alpha}$, т. е. чтобы ТР работали с равными опережающими и запаздывающими углами относительно тиристоров общей группы. Для косинусного $a_{v}$ и импульсного $b_{v}$ коэффициентов Фурье могут быть записаны

$$
\begin{aligned}
& a_{v}^{\prime}=\frac{1}{\pi v}\left\{-\int_{\alpha_{0}-\Delta_{\alpha}}^{\alpha_{0}} \cos v \omega t d \omega t+\int_{2 \pi / 3+\left(\alpha_{0}-\Delta_{\alpha}\right)}^{2 \pi / 3+\alpha_{0}} \cos v \omega t d \omega t\right\}, \\
& b_{v}^{\prime}=\frac{1}{\pi v}\left\{-\int_{\alpha_{0}-\Delta_{\alpha}^{\prime}}^{\alpha_{0}} \sin v \omega t d \omega t+\int_{2 \pi / 3+\left(\alpha_{0}-\Delta_{\alpha}\right)}^{2 \pi / 3+\alpha_{0}} \sin v \omega t d \omega t\right\}, \\
& a_{v}^{\prime \prime}=\frac{1}{\pi v}\left\{\int_{\alpha_{0}}^{\alpha_{0}+\Delta_{\alpha}} \cos v \omega t d \omega t-\int_{2 \pi / 3+\alpha_{0}}^{2 \pi / 3+\left(\alpha_{0}+\Delta_{\alpha}\right)} \cos v \omega t d \omega t\right\}, \\
& b_{v}^{\prime \prime}=\frac{1}{\pi v}\left\{\int_{\alpha_{0}}^{\alpha_{0}+\Delta_{\alpha}} \sin v \omega t d \omega t-\int_{\left.2 \pi / 3+\alpha_{0}+\alpha_{\alpha}\right)}^{1} \sin v \omega t d \omega t\right\},
\end{aligned}
$$

откуда после преобразования и учета значений тригонометрических функций при углах, кратных $2 \pi / 3$, можно найти их значения. Для опережающего ТР $\alpha^{\prime}=\alpha_{0}-\Delta_{\alpha}$

$$
a_{2}^{\prime}=\left\{-\sqrt{3}\left[\cos 2 \alpha_{0}-\cos 2\left(\alpha_{0}-\Delta_{\alpha}\right)\right]-3\left[\sin 2 \alpha_{0}-\sin 2\left(\alpha_{0}-\Delta_{\alpha}\right)\right]\right\} / 4 \pi,
$$

$b_{2}^{\prime}=\left\{3\left[\cos 2 \alpha_{0}-\cos 2\left(\alpha_{0}-\Delta_{\alpha}\right)\right]-\sqrt{3}\left[\sin 2 \alpha_{0}-\sin 2\left(\alpha_{0}-\Delta_{\alpha}\right)\right]\right\} / 4 \pi$,

$a_{4}^{\prime}=\left\{\sqrt{3}\left[\cos 4 \alpha_{0}-\cos 4\left(\alpha_{0}-\Delta_{\alpha}\right)\right]-3\left[\sin 2 \alpha_{0}-\sin 2\left(\alpha_{0}-\Delta_{\alpha}\right)\right]\right\} / 8 \pi$,

$b_{4}^{\prime}=\left\{3\left[\cos 4 \alpha_{0}-\cos 4\left(\alpha_{0}-\Delta_{\alpha}\right)\right]-\sqrt{3}\left[\sin 4 \alpha_{0}-\sin 4\left(\alpha_{0}-\Delta_{\alpha}\right)\right]\right\} / 8 \pi$.

Для запаздывающего ТP $\alpha^{\prime \prime}=\alpha_{0}+\Delta_{\alpha}$

$a_{2}^{\prime \prime}=\left\{-\sqrt{3}\left[\cos 2 \alpha_{0}-\cos 2\left(\alpha_{0}+\Delta_{\alpha}\right)\right]-3\left[\sin 2 \alpha_{0}-\sin 2\left(\alpha_{0}+\Delta_{\alpha}\right)\right]\right\} / 4 \pi$,

$b_{2}^{\prime \prime}=\left\{3\left[\cos 2 \alpha_{0}-\cos 2\left(\alpha_{0}+\Delta_{\alpha}\right)\right]-\sqrt{3}\left[\sin 2 \alpha_{0}-\sin \left(\alpha_{0}+\Delta_{\alpha}\right)\right]\right\} / 4 \pi$, 


$$
\begin{aligned}
& a_{4}^{\prime \prime}=\left\{\sqrt{3}\left[\cos 4 \alpha_{0}-\cos 4\left(\alpha_{0}+\Delta_{\alpha}\right)\right]-3\left[\sin 4 \alpha_{0}-\sin 4\left(\alpha_{0}+\Delta_{\alpha}\right)\right]\right\} / 8 \pi \\
& b_{4}^{\prime \prime}=\left\{3\left[\cos 4 \alpha_{0}-\cos 4\left(\alpha_{0}+\Delta_{\alpha}\right)\right]+\sqrt{3}\left[\sin 4 \alpha_{0}-\sin 4\left(\alpha_{0}+\Delta_{\alpha}\right)\right]\right\} / 8 \pi .
\end{aligned}
$$

Составляющие суммарного входного тока системы- образуются как суммы соответствующих составляющих опережающего и запаздывающего ТP

$$
\begin{aligned}
& a_{2}=a_{2}^{\prime}-a_{2}^{\prime \prime}=-\left(1-\cos 2 \Delta_{\alpha}\right)\left(\sqrt{3} \cos 2 \alpha_{0}+3 \sin 2 \alpha_{0}\right) / 2 \pi \\
& b_{2}=b_{2}^{\prime}+b_{2}^{\prime \prime}=-\left(1-\cos 2 \Delta_{\alpha}\right)\left(\sqrt{3} \sin 2 \alpha_{0}-3 \cos 2 \alpha_{0}\right) / 2 \pi \\
& a_{4}=a_{4}^{\prime}+a_{4}^{\prime \prime}=\left(1-\cos 4 \Delta_{\alpha}\right)\left(\sqrt{3} \cos 4 \alpha_{0}-3 \sin 4 \alpha_{0}\right) / 4 \pi \\
& b_{4}=b_{4}^{\prime}+b_{4}^{\prime \prime}=\left(1-\cos 4 \Delta_{\alpha}\right)\left(3 \cos 4 \alpha_{0}+\sqrt{3} \sin 4 \alpha_{0}\right) / 4 \pi
\end{aligned}
$$

По $a_{2}, b_{2}$ и $a_{4}, b_{4}$ найдем комплексные коэффициенты Фурье, которые характеризуют процентное содержание соответствующих составляющих в токе

$$
\begin{aligned}
& c_{2}=\sqrt{3}\left(1-\cos 2 \Delta_{\alpha}\right) / \pi, \\
& c_{4}=\sqrt{3}\left(1-\cos 4 \Delta_{\alpha}\right) / 2 \pi .
\end{aligned}
$$

Графики функций $c_{2}\left(\Delta_{\alpha}\right)$ и $c_{4}\left(\Delta_{\alpha}\right)$. приведены на рис. 4. Подчеркнем их основные свойства.

1. В рассматриваемой системе при конечных отличиях углов управления нельзя достичь нулевого значения четных гармонических составляющих входного тока. Однако их подавление в сравнении с подавлением в системе на общих диодах будет значительным. Ниже это иллюстрируется примером. Их подавление достигается также, если сравнить с таковым в $\left[{ }^{3}\right]$.

2. Эффект подавления четных гармонических составляющих инвариантен относительно абсолютных значений углов управления.

Проиллюстрируем достигаемый эффект примером с использованием вышеприведенных данных.

I. В системе с общей диодной группой коэффициент 1-й гармонической составляющей каждого из ТР составляет $c_{1}^{\prime}=c_{2}^{\prime \prime}=$ $=\frac{\sqrt{6}}{\pi} \sqrt{1+\cos \frac{\pi}{2}}=0,7795$. Их сумма во входном токе составляет $c_{1}=c^{\prime}{ }_{1}+c_{1}{ }^{\prime \prime}=1,559$. По графику приведенной функции $v \frac{c_{v}}{c_{1}} \quad$ в $\left[{ }^{5}\right]$ можно найти в рабочей точке при $\alpha^{\prime}=\alpha^{\prime \prime}=\pi / 2 \quad c_{2}=0,75 \cdot 1,559=1,17$.

II. В группе, сравнимой с общей произвольно-управляемой тиристорной группой при $\Delta_{\alpha}=13^{\circ}$, по рис. 4 найдем $c_{2}=0,06$. Соотношение токов второй гармонической составляющей $I_{100}^{\mathrm{I}} / I_{100}^{\mathrm{II}}$ равно $1,17 / 0,06=19,5$. Другими словами, при переходе к системе с общей произвольно-управляемой тиристорной группой достигаем существенного эффекта подавления четных гармонических составляющих входного тока низкого порядка. Экспериментальная проверка подтвердила полученный результат (измеренные на спектроанализаторе со встроенным микрокомпьютером данные показаны на рис. 4 ноликами для 2 -й и крестиками для 4-й гармонических составляющих). 
Д̆ля подавления четных гармонических составляющих в совокупности или в отдельности при неравных токах целесообразно отказаться от жесткого произвольного управления тиристорами общей группы, а формировать последовательность управляющих импульсов $\alpha_{0}=\mathrm{var}$ в зависимости от результата гармонического анализа. В этих целях может быть построена система управления на базе микропроцессорной техники, так как управление по $\alpha_{0}$ может быть медленно действующим в отличие от систем управления ТР, которые должны формировать последовательности $\alpha^{\prime}, \alpha^{\prime \prime}$ в реальном масштабе времени.

\section{Выводы}

Система с общей произвольно-управляемой группой тиристоров имеет большие преимущества перед системой с общей диодной группой и определенные преимущества перед системой с общей группой тиристоров, управляемых с минимальным в системе углом управления. Для нагрузок с постоянным напряжением достаточно применение фиксированного угла управления общих тиристоров, в более сложных случаях целесообразно применять функциональное управление общими тирнсторами.

\section{Л И Т Е РА Т У Р А}

1. Mc Murray, W. IEEE Trans. Ind. Appl., 1A-8, № 3 (May/June), 289-295 (1972).

2. Томсон Т. И. А.с. СССР по заявке № $3797680 / 24-07$. Положительное решение от 3. 10. 1985.

3. Томсон Т. Изв. АН ЭССР. Физ. Матем., 35, № 1, 93-105 (1986).

4. Томсон T. Изв. АН ЭССР. Физ. Матем., 34, № 1, 93-106 (1985).

5. Томсон T. Изв. АН ЭССР. Физ. Матем., 33, № 3, 339-350 (1984).
Институт термофизики и электрофизики Академии наук Әстонской ССР
Поступила в редакцию 6/VIII 1986

\section{T. TOMSON}

\section{SUVALISELT JUHITAVATE OHISTURISTORIDEGA IDEALISEERITUD ALALDITE SUSTEEM}

On selgitatud ühise, suvaliselt juhitâvva türistorirühmaga türistoralaldite süsteemi omadused väljundpingete ja sisendvoolu harmoonilise koosseisu poolest. On tõestatud, et autonoomsete türistoride sümmeetriliselt hilineva ja ennetuva juhtimisimpulsi puhul saadakse ebasoovitavate harmooniliste komponentide oluline mahasurumine, vôrreldes analoogilise, kuid ühisdioodidega süsteemiga.

\section{T. TOMSON}

\section{THE IDEALIZED RECTIFIER SYSTEM WITH ARBITRARILY CONTROLLED COMMON THYRISTORS}

The characteristics of a rectifier system with common arbitrarily controlled thyristors are determined in terms of the harmonic composition of output voltages and the input current. It has been shown that while controlling autonomous thyristor groups by symmetrically advanced and delayed pulses, we get significant suppression of unwanted harmonics compared to the analogous system with a common diode group. 